\title{
Qualitative Evaluation of Physical Effort in Bass Drum Pedal Drive by Thermography
}

\author{
Cláudio L. Salvalaio ${ }^{1, *}$, Fábio P. Silva ${ }^{2}$, Alexandre S. Pinho ${ }^{3}$, Mariana Pohlmann ${ }^{4}$ \\ ${ }^{1}$ Laboratory of Design and Materials Selection (LdSM), UFRGS, Porto Alegre, 90035-190, Brazil \\ ${ }^{2}$ DEG, UFRGS, Porto Alegre, 90035-190, Brazil \\ ${ }^{3}$ Department of Biomechanics, IBTeC, Novo Hamburgo, 93334-000, Brazil \\ ${ }^{4}$ LdSM, UFRGS, Porto Alegre, 90035-190, Brazil
}

\begin{abstract}
The bass drum is driven by a pedal that promotes muscle fatigue and can cause injuries. In order to provide engineers with valuable tools, this research aimed at analyzing the use of the drum pedal and to evaluate qualitatively the muscle groups involved and their possible relationship with any injuries resulting from fatigue. Through the analysis, it was found that striking the drum pedal provides health risks; therefore, some actions were suggested to avoid injuries to drummers. All data collected can be used as parameters for the development of products that would prevent health problems or improve the drummers' performance.
\end{abstract}

Keywords Bass Drum Pedal, Fatigue, Thermography

\section{Introduction}

In music, dynamics is the variation of sound intensity and when combined with other features can provide great expressiveness to music. These variations distinguish an excessively precise mechanical execution of an interpretation that conveys the emotions planned by the composer[1]. Intensity refers to the ability of a sound to be stronger or weaker than the other, it is the accent, the emphasis on a sound. Dynamic is largely worked in music by acoustic drums. This consists of a set of drums and cymbals played by a musician named drummer. Among the pieces that comprise this set, the bass drum is driven by a mechanical pedal. In 1950, in the United States, the first record of the musical style, Rock'n'roll, was released[2]. Heavy Metal was derived from Rock'n'roll and appeared in the 60 's characterized by a heavy, fast and virtuous sound[3].

The emergence of these new musical styles with strong speed characteristic required adjustments, new equipment and the development of the instrumentalist's highly technical/practical skills. The pedal body is made of light metal alloy, the spring system of the beater return was improved, and some regulations were included for adjustments and the axis friction was reduced by bearings. For speed, to increase the number of beats per minute on a

* Corresponding author:

claudiosalvalaio@gmail.com (Cláudio L. Salvalaio)

Published online at http://journal.sapub.org/scit

Copyright (C 2011 Scientific \& Academic Publishing. All Rights Reserved bass drum, a drive shaft with another pedal was added, creating the double pedal (Figure 1) which is driven by both feet. All these improvements have made the drive fast and smooth.

Drummerworld[4] observed that some drummers reach 280 beats per minute in the bass drum with the double pedal. In a concert, a drummer may use the pedal for about one hour with only brief intervals between songs. Using arithmetic average, there is about 10.000 hits on the bass drum.

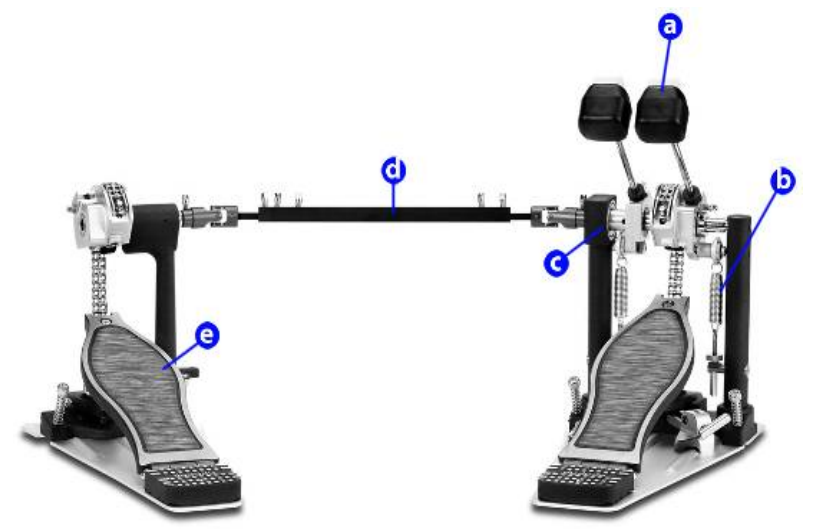

a) beater $\cdot$ b) spring $\cdot$ c) axis $\cdot$ d) drive shaft $\cdot$ c) footboard

Figure 1. Bass drum double pedal.

Burke[5] reported that researchers at the University of Chichester and Gloucestershire, England, made an eight-year experience with a drummer by installing equipments to measure the heart rate, oxygen consumption and lactic acid levels in the blood. Experts have concluded 
that playing drums takes a lot of energy and activates the aerobic and anaerobic systems in the organism. Even with the improvements implemented in the pedal, contrary forces act against the drive: the spring tension and the friction of the axes. The effort to overcome these forces also causes fatigue, making the drive a risk to the user. Thus, the pedal imposes a mechanical limit to the drummer and not a physical one, which could be exceeded. The current conception referring to pedals restricts the drummer to a single position in front of the drum set, reducing the percentile of individuals who could play this instrument. Considering these aspects, even a slight limitation in the lower limbs is a factor that may restrain this activity. The effort and speed combination to drive the pedal is very stressful when exacerbated by long periods of repetitive activity, could cause injury to muscles and joints[6].

Tendonitis first symptoms are pain on exertion; this pain often radiates to other parts of the limb. When the tendon inflammation is more intense, swelling at the site may occur after effort. If untreated, tendinitis can progress to numbness and tingling sensations, limb generalized swelling, frequent and intense pain, decrease of endurance, muscle strength and motor skills affecting the musician performance. Once the disease is installed, the person is unable to perform some activities, which can lower his/her self-esteem. According to Vash[7], undeveloped or damaged self-esteem can cause psychiatric disorders.

In order to provide designers and engineers with tools in product design, this research analyzed the use of bass drum pedal by the drummers and evaluated qualitatively, using the thermography technique, the muscle groups involved and their possible relationship with any injuries resulting from fatigue. Thus, the data collected can serve as a parameter for the suitability of pedal drive techniques and for the development of new products such as shoes, drum pedals or systems to reduce health risks and improve the users' performance.

\section{Material and Methods}

For an effective result in the evaluation of muscular effort in pedal drive in its current state of the art technique, the following methods were used: questionnaires, ergonomic analysis, force measurement and temperature analysis by thermography.

A 15-question multiple choice questionnaire related to a specific action while using the pedal was applied to thirty-eight Brazilian drummers. In the pedal ergonomic analysis, some aspects were evaluated such as safety use, equipment transportation, anthropometry (relating to the measures between product and user) and cognition (user perception related to the product and its use).

Anthropometric data are often expressed in percentile terms. According to Panero[8], percentiles indicate the individual percentage within a population that has a certain body size or smaller. For study purposes, the population is divided into 100 percentage categories from the highest to the lowest for any specific type of body measurement. The percentile 95 for height, for example, indicates that only $5 \%$ of the population has greater heights. Since it becomes difficult to do research and to design for the entire population, it is necessary to select a central portion segment. Therefore, it is customary to work with $90 \%$ of the population group in question without considering the extremes at both ends.

To survey the task activities and postural assessment, the position and movements of two drummers were analyzed, a 5 th percentile woman to a 95 th percentile man (Table 1).

Table 1. Anthropometric data on adult height and weight[8].

\begin{tabular}{|c|c|c|c|c|}
\hline Percentile & Gender & Age & Weight $(\mathrm{kg})$ & Height $(\mathrm{m})$ \\
\hline 5 & Female & $18-24$ & 44.9 & 1.52 \\
\hline 95 & Male & $18-24$ & 97.1 & 1.85 \\
\hline
\end{tabular}

Panero[8] defined the range of joint motion as the following: for the hip, $45^{\circ}$ on hyperextension and $120^{\circ}$ on flexion, total $165^{\circ}$; for the flexed knee $135^{\circ}$ and $16^{\circ}$ on hyperextension, total $141^{\circ}$; for ankle joint in relation to the leg, $70^{\circ}$ on dorsiflexion and $125^{\circ}$ on plantarflexion.

The force measurement on beater and muscle effort aimed at measuring the force reached in the pedal beater. Data collection was obtained from a drummer by using a dynamometer, which measures mechanical force through a spring coupled to his body. Through the spring deformation, it is possible to get the amount of force that caused it (though this is a slow kind of instrument for static or dynamic measurement). The dynamometer was fastened on a metal structure and attached to the beater rod. A similar test was conducted by drumming the pedal's beater against an analog scale fixed to the metal structure. This kind of measurement is not standardized, and it is difficult to assess other variables such as the user's physical/muscular fitness and the environment. It is considered that values extracted by the analysis can be used satisfactorily as an estimate for forces with which the pedal works.

For the description of the involved muscles in the pedal drive, the evaluation of the body temperatures was carried out in the lower limbs. Thermography is a noninvasive diagnostic method that measures and displays the emission of infrared radiation applied in proportion to the body surface temperature, related to the skin blood flow[9]. The thermography is an image generated by infrared rays that are emitted from a surface. Malignancies, inflammation, infection and other abnormalities can increase localized temperature in tissue. In the thermographic image, this appears as hot spots or areas of inhomogeneity. It has been proven reliable and convenient as it has been used to investigate a variety of clinical problems. One of the applications of the thermography is to evaluate inflammatory diseases, such as rheumatic disease of the hands and osteoarthritis of the knees and hands. Some studies reported that the arthritis activity and severity indices can be indicated by thermal measurements[10]. 
The individual evaluated was a 30 year old drum teacher, 11 years of experience, right-handed, height $1.79 \mathrm{~m}$, weight $85 \mathrm{~kg}$. Two techniques were analyzed with the use of the pedal: Heel Toe, a more natural technique to the user with the bass drum being driven once per pedal. The heel remains in contact with the pedal and the movements are performed mainly in the ankle joint (initial position: ankle on dorsiflexion; end position: neutral ankle); Toe Pivot, heel is raised and the toe touches the pedal. It requires more control as the leg rotates and two beats per pedal are performed (starting position: hip and knee flexion, neutral ankle and foot eversion; end position: hip and knee extension, neutral ankle and foot inversion). The resources used in this analysis were: double pedal Iron Cobra HP900PTW - TamaC, electronic drum bass pad STD90 - Shelter $\bigcirc$, electronic metronome GMT-200P - Groovin(C, stool T575A selim type - Mapex $($, headset HP250 - Philips $\subseteq$, thermograph PV320-T2 - Eletrophysics $\odot$.

\section{Results and Discussion}

\subsection{Questionnaire}

Thirty-eight drummers, average age 28 years old, height $1.74 \mathrm{~m}$ and weight $75.5 \mathrm{~kg}$, answered the questionnaire, thirty-seven were male (Table 2).

Table 2. Sampling of drummers.

\begin{tabular}{|c|c|c|c|c|}
\hline & Age & $\begin{array}{c}\text { Height } \\
(\mathbf{m})\end{array}$ & $\begin{array}{c}\text { Weight } \\
(\mathbf{k g})\end{array}$ & CMI \\
\hline Average & 28 & 1.74 & 75.5 & 24.07 \\
\hline $\begin{array}{c}\text { Standard } \\
\text { deviation }\end{array}$ & $+/-3.3$ & $+/-14.5$ & $+/-9.8$ & $\begin{array}{c}+/- \\
12.1\end{array}$ \\
\hline Min value & 10 & 1.51 & 51 & 22.37 \\
\hline Max value & 46 & 1.97 & 100 & 25.77 \\
\hline
\end{tabular}

Among the respondents, $40 \%$ have been playing drums for over 6 years, $26 \%$ between 4 and 6 years, $21 \%$ between 1 and 3 years and $13 \%$ for less than 1 year. Regarding the use of the pedal, $53 \%$ use double pedal and $47 \%$ use single pedal. Among the light, medium and heavy levels of effort to drive the pedal, $61 \%$ considered medium, $34 \%$ light and $5 \%$ considered heavy. The percentage of respondents who felt tired after 2 hours of playing is $42 \%, 29 \%$ after 1 hour and $16 \%$ after 3 hours. With regards to the total drummers, $66 \%$ felt fatigue in the legs (thigh and calf), $17 \%$ in the back, and $8 \%$ in the feet (Figure 2). On the same graph, projections indicated combined fatigue: legs and feet $8 \%$, legs and back $8 \%$.

For $16 \%$ of the respondents, tiredness persists until the next day with some muscle pain. Nevertheless, 55\% had never thought that bass drum drive could cause Repetitive Stress Injury, 37\% thought it did not cause and $8 \%$ thought it might cause. Reflecting on the condition of muscle stress and fatigue after bass drum drive, $79 \%$ of the drummers show interest in using a lighter drive pedal and $84 \%$ of them in using a pedal that could provide higher speed drive.
Among the total number of respondents, $71 \%$ considered important the development of a much free pedal which can be driven with the feet, legs and even arms, besides enabling the instrument practice to injured/disabled (lower limbs) individuals.

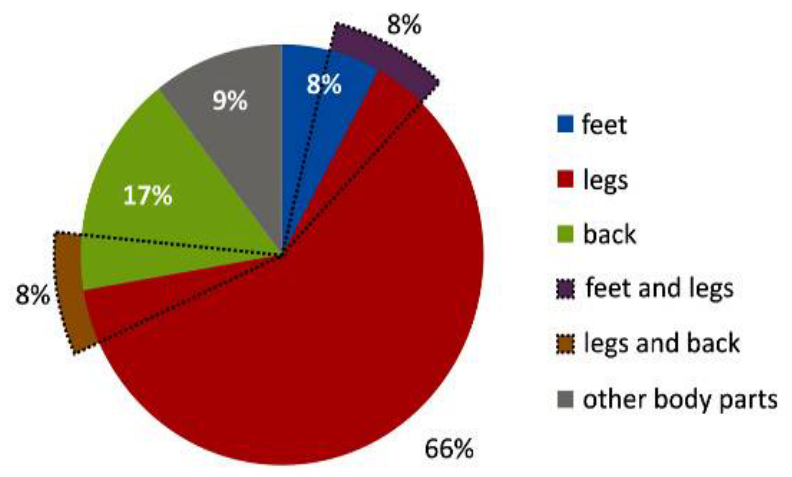

Figure 2. Muscular fatigue sensation on pedal drive..

An item for a free comment was inserted at the end of the questionnaire, in which some drummers have expressed their opinions. Below, some of these reviews are presented:

- "I have difficulty in some pedal techniques because my foot is relatively large and some footboards are small, especially when I use mid foot to heel".

- "I wear 11/12 and I feel that the footboard is a bit small for my foot. I think the idea of a long board is interesting. A pedal must be reliable; you will probably not buy a pedal that looks like it will break at the first song".

- "Perhaps a study on the sitting posture while playing should be developed to minimize injuries and fatigue".

\subsection{Ergonomic Analysis}

Regarding safety, the pedal can bring a crushing risk, especially of the fingers if any part of the body is improperly inserted in the pedal drive region. It is made of metal and has sharp edges which can hurt the user when he/she strikes the pedal. For transportation, the double pedal disengagement becomes easier, but there is no handle or strap to carry it. Anthropometrically, when in use, only the foot remains in contact with the one-size fits all pedal shoe and some drummers have problems with this regard. There is a pedal height adjustment that prevents improper touch with the foot in the drive system. Cognitively, the pedal is simple and used intuitively, only the settings require greater knowledge.

By analyzing the task activities, it can be observed that the pedal is fixed to the bass drum without the use of tools. A stool used to sit in front of the drum rotates; it has adjustable height and is upholstered. There is backboard option, but is not widely used. As the backboard is not used, this seat can cause physical discomfort to the user, in that case, according to [8], the body instability increases and muscle strength is needed for additional balance. The drummer sits at the stool, sets his feet on the pedal and drives the bass drum by the beater. Arms and hands are involved in playing rack toms, snare drums and cymbals. When the pedal is driven, the hip, knee and ankle joints move. To achieve speed with a 
minimum of muscular effort, these movements should be made smoothly. In the questionnaire, it was shown that the pedal drive caused back, leg and feet fatigue. While evaluating the activity, it was determined that back fatigue is from the lower limbs movement with dangling feet. Leg fatigue is due to the pedal drive plus feet lifting that alternate the movements almost without any support. Feet fatigue occurs by moving and lifting the feet

In postural evaluation, angles and range of motion were identified in two drive pedal techniques between two users, one female and one male, in 5 and 95 the percentile, respectively. In Heel Toe technique, the woman (Figure 3) showed a $17^{\circ}$ variation in the ankle joint and the man (Figure $4)$, a $13^{\circ}$ variation in the same joint.

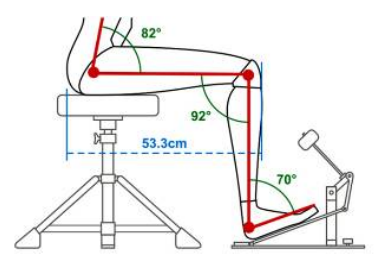

a)

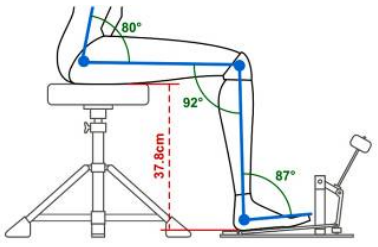

b)
Figure 3. Woman joint angle variation in Heel Toe, a) initial position and b) end position.

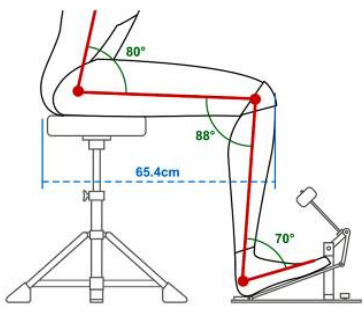

a)

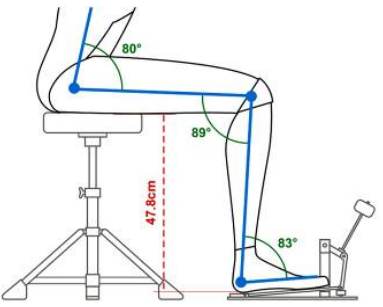

b)
Figure 4. Man joint angle variation in Heel Toe, a) initial position and b) end position.

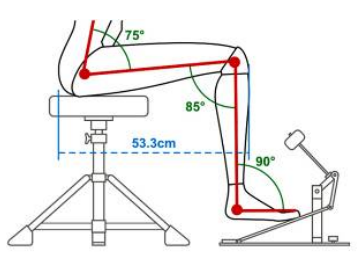

a)

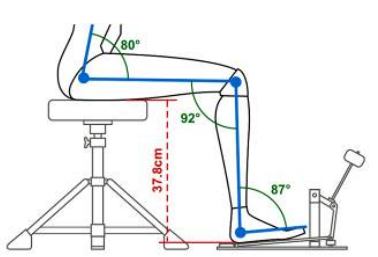

b)
Figure 5. Woman joint angle variation in Toe Pivot, a) initial position e b) end position.

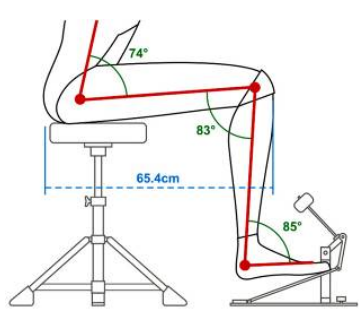

a)

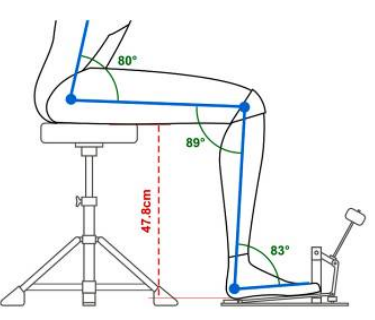

Figure 6. Man joint angle variation in Toe Pivot, a) initial position e b) end position.

\subsection{Beater Force and Muscular Effort Measurements}

In an attempt to apply constant weight in three bands called maximum, medium and minimum, drummer reached the maximum weight of $50 \mathrm{~N}$ (Newtons) measured by the dynamometer (Figure 7a), the medium weight was between $20 \mathrm{~N}$ and $30 \mathrm{~N}$ and the minimum, $5 \mathrm{~N}$. With the scale (Figure $7 \mathrm{~b})$, the maximum weight was $4 \mathrm{~kg}$ (kilogram), the medium weight was between $1 \mathrm{~kg}$ and $2 \mathrm{~kg}$ and the minimum, $0.5 \mathrm{~kg}$.
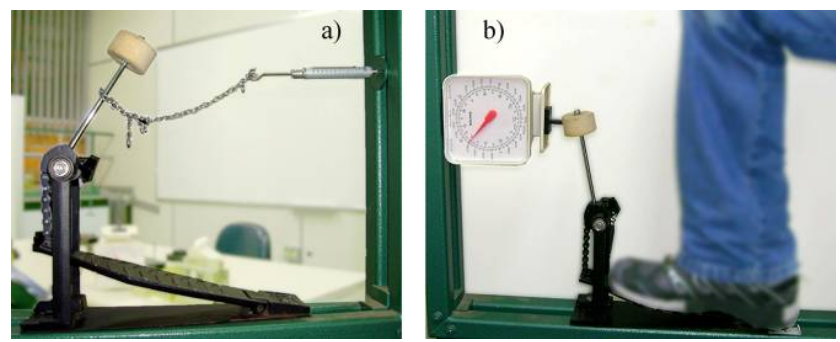

Figure 7. Measurement of force with dynamometer (a) and scale (b).

To work the dynamics in music and to gain variations in sound intensity, it is necessary to use different muscle forces in the bass drum drive. This will increase the muscular effort at certain moments during the instrument execution. For strong sounds in drum bass, the level of muscular effort required in driving the pedal can be considered heavy and for weak sounds, light. With the force measurement analysis, it was possible to establish this parallel between the force in the beater and the muscular effort for pedal drive (Table 3).

Table 3. Parallel between physical effort and force in the beater.

\begin{tabular}{|c|c|c|}
\hline Force on beater & $\begin{array}{c}\text { Average value between } \\
\text { dynamometer and scale }\end{array}$ & Muscle effort \\
\hline Max & $45 \mathrm{~N}$ & Heavy \\
\hline Medium & $20 \mathrm{~N}$ & Moderate \\
\hline Min & $5 \mathrm{~N}$ & Light \\
\hline
\end{tabular}

\subsection{Thermograph Evaluation of the Muscles Involved}

The protocol for thermograph evaluation of muscle temperature (Figure 8 ) followed this procedure: the drummer remained for 30 minutes at rest and then the first capture was made with a thermographic camera. After a brief stretch, without heating, the drummer started to play following the Heel Toe technique. The speed was defined as the average applied in rock music, marked by a metronome at $80 \mathrm{bpm}$, resulting in 160 beats per minute due to the double pedal.

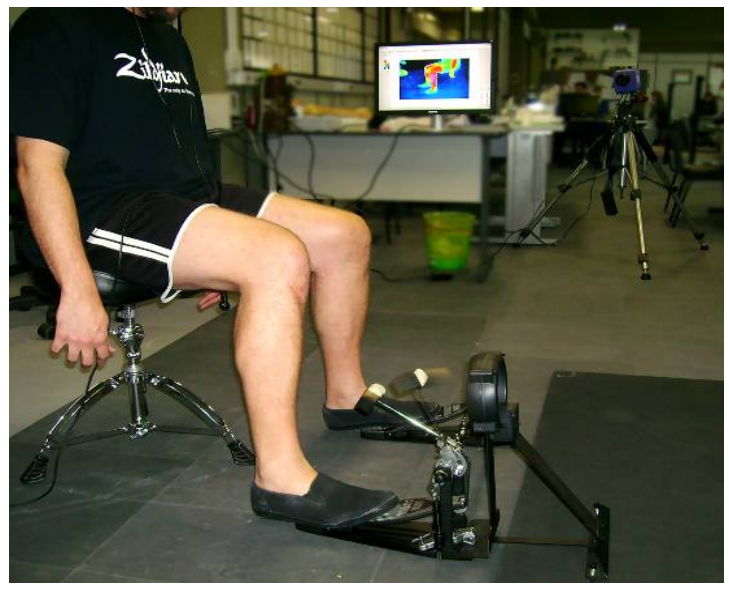

Figure 8. Thermography evaluation of muscular temperature. 
Captures occurred at 10 minute intervals of action: the first at rest, the second after 10 min playing and the third after $20 \mathrm{~min}$. For the Toe Pivot technique, the drummer took a 45 minute break to return to the resting temperature and the same capture sequence of the former technique was adopted. In Figure 9, the evolution of the muscle temperature with the Heel Toe technique in relation to time (and hence muscle activity) was observed. In thermography color scale, black indicates the lowest temperatures and violet the highest.
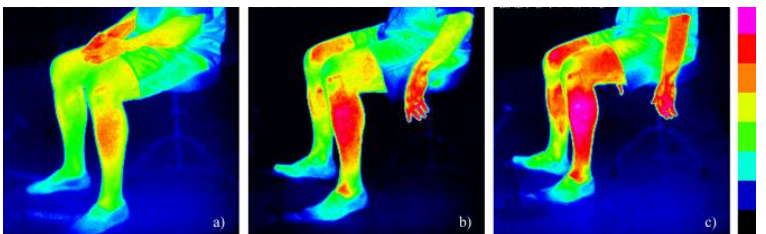

Figure 9. Heel Toe technique - At rest (a), after 10min playing (b) and after $20 \mathrm{~min}$ playing (c).

In Figure 10, the evolution of the drummer muscular effort using the Toe Pivot technique with bass drum drive, double pedal can be observed.
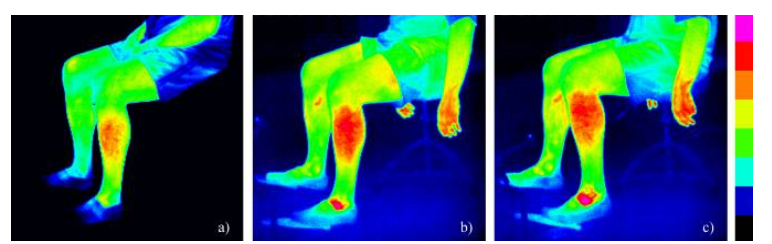

Figure 10. Toe Pivot technique - At rest (a), after 10min playing (b) and after $20 \mathrm{~min}$ playing (c).

In Figure 11, the regions of higher muscle temperature in Heel Toe (a) and Toe Pivot (b) techniques are shown; both thermographic images refer to 20 minutes of action.
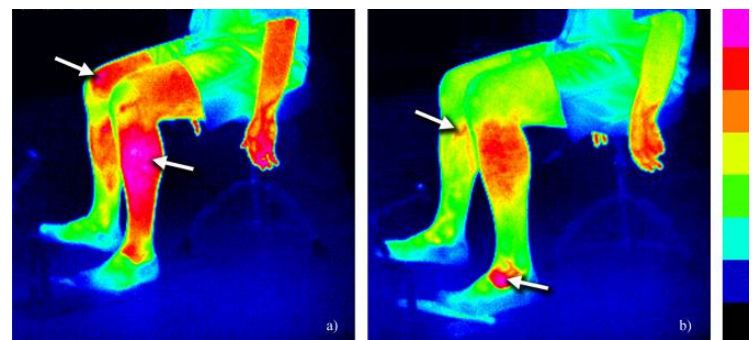

Figure 11. Areas of increased muscle temperature (see the arrows), Heel Toe (a) and Toe Pivot (b).

For a complete analysis of the lower limb muscles involved, the thermographic images were captured from the front and back in both techniques at 20 minutes of playing. In Figure 12, the Heel Toe technique, the largest areas of heating are shown.

By thermography, it can be observed that in the Heel Toe technique the most requested muscles are ankle dorsiflexors (tibialis anterior, extensor digitorum longus and peroneus tertius) and knee extensors (quadriceps). In this technique, the areas of higher temperature (indicated by arrows in Figure 11a) are concentrated in the lateral portion of the leg, medial knee and thigh, being associated with greater muscle activity.
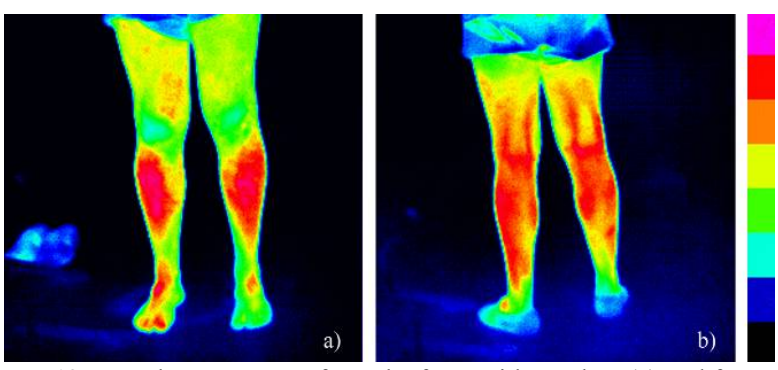

Figure 12. Heel Toe: capture from the front without shoe (a) and from the back (b).

In Toe Pivot technique, Figure 13, there is higher and more punctual warming in the right limb, indicating that the drummer uses it more, for being right-handed probably.
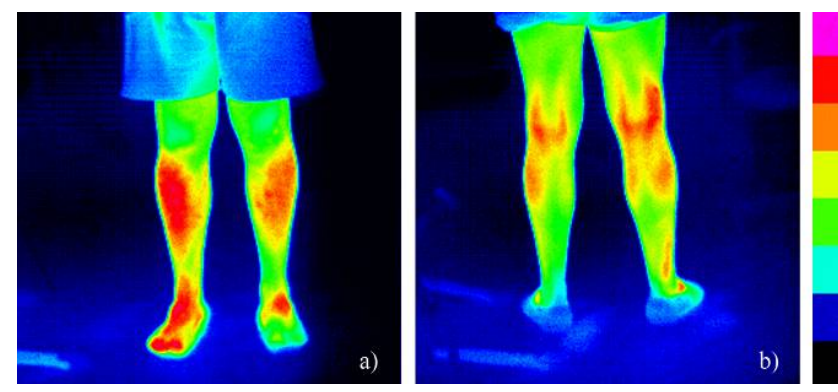

Figure 13. Toe Pivot: capture from the front without shoe (a) and from the back (b).

To drive the bass drum pedal almost the same muscles (Figure 14) are required in both techniques, the ankle dorsiflexors: tibialis anterior, extensor digitorum longus and peroneus tertius. In the Heel Toe technique, the knee extensors are also involved: vastus lateralis, vastus medialis, vastus intermedius and rectus femoris (quadriceps). This technique produces higher heating (probably due to an increased muscle activity) and covers more muscular areas. In Toe Pivot, there is effort concentration especially in the ankle, and in an isolated point at the medial knee portion due to rotation. It is likely that, with prolonged use, both techniques may cause injury, but the Toe Pivot technique is more likely to cause Repetitive Stress Injury due to a mechanical concentration at localized points. a)

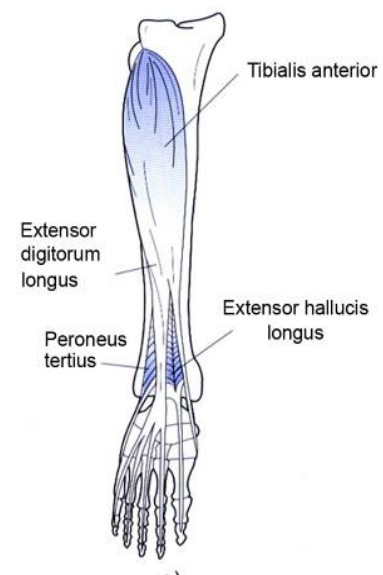

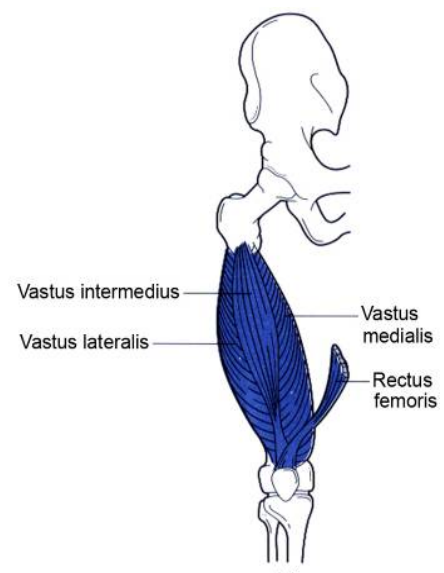

b)
Figure 14. Muscles: Ankle dorsiflexors (a) and knee extensors (b)[12]. 


\section{Conclusions}

The pedal restricts the drummer's position in front of the instrument. This lack of mobility can drastically increase the chance of developing a repetitive motion injury. According to research, literature in the field, it was verified that the pedal shape and aesthetics are less relevant in relation to the quality characteristics of the bass drum drive, such as lightness, precision and speed. In the ergonomic analysis, it was observed that the drummers' adopted position is consistent with the joint angles suggested by Panero[8]. With these data, the chances that the lower limbs fatigue and pain (reported by the users in the questionnaire) can be the result of poor posture are greatly reduced. By thermography, the areas of higher temperature were mapped (associated with greater muscle activity), in which there was a greater probability of injury due to the pedal prolonged use.

The technique, in which the foot works in neutral position ranging a few degrees, is used to achieve the bass drum driving speed. It applies to the rebound, when the pedal is activated in two stages on the same ride. The joints with wider range of operation are the knee and hip. This technique requires additional lower limbs muscles effort to keep the feet from hanging over the pedal. Among the techniques studied, the one with the foot in dorsiflexion is more likely to cause Repetitive Stress Injury, as it works within the range limits of the analyzed motion. The foot in neutral position technique may fatigue all the muscles. According to Burke[5], playing drums is an intense and physically demanding activity.

Therefore, it appears that pedal can cause muscle fatigue and eventually generate Repetitive Stress Injury which will limit or even prevent the musician from playing. As prevention and corroborating Lima[11], the drummer should perform more naturally while activating the joints, stretch and warm up the muscles before playing, take some rest periods during the activity to avoid excessive repetitive movement, educate the posture, keep on a regular physical activity to allow tendons to stretch, lengthen and relax, look for a qualified professional to the slightest hint of a tendonitis. The use of appropriate footwear can reduce the points of concentrated stress through the distribution and increase of the muscle area involved in the movement.

This research could serve as a basis for the suitability of the pedal drive technique, the development of appropriate footwear as well as the development of new products which will certainly reduce the health risks and improve the drummer performance.

\section{ACKNOWLEDGEMENTS}

The authors would like to thank the contribution provided by the Brazilian Institute of Technology for Leather and Footwear (IBTeC), Lab Design and Material Selection of Federal University of Rio Grande do Sul (LdSM/UFRGS), Ph.D. Milton Antônio Zaro, Ph.D. Wilson Kindlein Júnior, M.Sc. Gustavo Freitas, M.Sc. Airton Luis Kleinowski, Drummer Marcelo Dornel Gevehr, Bel. Yuri Bruxel.

\section{REFERENCES}

[1] Med, B. Teoria da Música. 4.ed. Brasília: Musimed, 1996.

[2] Montanari, V. História da música: da idade da pedra à idade do rock. 2.ed. São Paulo: Ática, 1992.

[3] Martin, G. The Illustrated Encyclopedia of Music: From Rock, Jazz, Blues and Hip Hop to Classical, Folk, World and More. Londres, UK: Flame Tree, 2003.

[4] Drummerworld. George Kollias: Intense Metal Drumming DVD. Available on: $<$ http://www.drummerworld.com/videos /georgekollias200280.html>. Accessed on: Oct 22, 2009.

[5] Burke, C. The energy cost of rock drumming: a case study. Available on: $<$ http://www.clemburkedrummingproject.com/ Research.html\#european>. Accessed on: May 2, 2011.

[6] Dul, J. Ergonomia prática. 2.ed. São Paulo: Edgard Blücher, 2004.

[7] Vash, C. L. Enfrentando a deficiência: a manifestação, a psicologia, a reabilitação. São Paulo: Pioneira/EDUSP, 1988.

[8] Panero, J. Zelnik, M. Las dimensiones humanas en los espacios interiores. Barcelona: GG, 2007.

[9] Christiansen, J., Gerow, G. Thermography: seminars in chiropractic. Lippincott Williams \& Wilkins, 1990.

[10] Chin L.W., Kwong, L.Y.; Hung, Y. C.; Mao, H.H.; Tien, W.C.; Chia, H.C. The application of infrared thermography in the assessment of patients with coccygodynia before and after manual therapy combined with diathermy. Journal of Manipulative and Physiological Therapeutics, Volume 32, Number 4, 2009.

[11] Lima, R. C. Tendinite: uma ameaça que pode ser evitada. Available on: $<\mathrm{http}: / / \mathrm{www} \cdot$ batera.com.br/artigos/tendinite_c uidados.aspx>. Accessed on: May 2, 2009.

[12] Guy, J.F. Learning Human Anatomy: A laboratory text and workbook. Ohio: Prentice Hall, 2009. 